\title{
Minimal coupling of electromagnetic fields in Riemann-Cartan space-times for perfect fluids with spin density
}

\author{
Larry L. Smalley \\ Department of Physics, University of Alabama, Huntsville, Alabama 35899 \\ Jean P. Krisch \\ Department of Physics, University of Michigan, Ann Arbor, Michigan 48109
}

(Received 18 July 1991; accepted for publication 16 October 1991)

\begin{abstract}
The electromagnetic field is minimally coupled to gravity in a Riemann-Cartan space-time containing a charged magnetized spinning fluid. It is required that the overall Lagrangian of the gravitational field, spinning matter, and the electromagnetic field be invariant under a gauge transformation of the vector potential. The theory preserves both charge conservation and particle number conservation. The electromagnetic field, via the vector potential, now interacts directly with the spin energy momentum. The spin transport equation, in addition to the usual Fermi-Walker transport term, contains a contribution due to the torque of the electromagnetic field acting on a magnetic dipole. In the absence of electromagnetism, the field equations reduce to those of the usual self-consistent Lagrangian formalism for a perfect fluid with spin density.
\end{abstract}

\section{INTRODUCTION}

We consider the problem of "minimally" coupling the electromagnetic (EM) field to a Riemann-Cartan (RC) space-time for charged fluids with spin density. One of the major historical problems with coupling the EM field to any space-time geometry has been the maintenance of gauge invariance or charge conservation in the theory. ${ }^{1}$ The most common prescription for lifting special relativity to general relativity (GR) is to replace ordinary derivatives with covariant derivatives. This is the principle of minimal coupling. When this principle is applied to electromagnetism, the special relativistic Maxwell field tensor,

$$
F_{i j}=A_{j, i}-A_{i, j}
$$

becomes

$$
F_{i j}=A_{j, i}-A_{i, j}
$$

In GR, the symmetric Christoffel connection renders these definitions equivalent.

The generalization of a geometrical object to a more complex space can be done in many ways; there is no $a$ priori requirement that the EM field be a two-form. ${ }^{2}$ One is free to impose prior constraints on the fields or overall constraints on the form of the field equations after generalization, for example, one may require that the Lagrangian satisfy certain conditions such as overall gauge invariance.

As an example of the above discussion, one extension of GR is the Einstein-Cartan (EC) theory in RC space-times. ${ }^{3}$ In this theory, space-time has a spin acti- vated torsional property so that the connection now contains torsion terms in addition to the GR Christoffel part

$$
\Gamma_{j k}^{i}=\left(\begin{array}{c}
i \\
j k
\end{array}\right)+S_{j k}^{i}-S_{k j}^{i}+S_{j k}^{i}
$$

where $S_{i j}{ }^{k}=\Gamma_{[i]}^{k}$. Because of the antisymmetry of the connection, there are ambiguities and difficulties in generalizing the principle of minimum coupling to the treatment of electromagnetism in the EC theory. ${ }^{2,4}$ The largest problem involves maintaining gauge invariance and therefore charge conservation. Two major approaches have been used to describe electromagnetism in RC space-times. One either defines the field tensor by Eq. (1), giving up minimal coupling in return for gauge invariance, or one enforces minimal coupling by using Eq. (2) and develops models to deal with the gauge invariance difficulties. Hehl ${ }^{5}$ has proposed using Eq. (1) for Maxwell fields, reserving minimal coupling for massive fields, like a Proca field. This suggestion will decouple photons from the spin-activated torsion. de Ritis et al. ${ }^{6}$ and Amorim ${ }^{7}$ have developed a charged spin fluid formalism in RC space-time assuming that the field tensor is a two-form given by Eq. (1). The simple use of Eq. (1) can also require the introduction of interaction terms into the Lagrangian, as in the work of De Sabbata and Gasperini. $^{8}$

Hojman et al. ${ }^{9}$ and Hammond, ${ }^{10}$ among others, have developed formalisms that enforce minimum coupling. In the work of Hojman, a propagating scalar field was introduced to help solve the gauge invariance problems. This field served to restrict the allowed form of the torsion and has as its source the EM field. The theory predicts the existence of both electric and magnetic currents. 
Mukki and Sajed ${ }^{11}$ extended this approach to Yang-Mills fields. In Hammond's approach to minimal coupling, ${ }^{10}$ the ordinary Einstein-Maxwell theory is interpreted as a RC gravitational field with propagating torsion. The torsion vector was assumed proportional to the electromagnetic vector potential $\boldsymbol{A}_{k}$. In this model, the photon develops a nonzero mass.

The purpose of this paper is to develop a minimally coupled Lagrangian theory that will treat magnetized and charged spin fluids in RC space-times without introducing massive photons, restricted torsion, or other unwanted features. This work is an extension of the selfconsistent Lagrangian for a perfect fluid with the spin density developed by Ray and Smalley. ${ }^{12}$

In our approach, the self-consistent Lagrangian formalism for spinning fluids in RC space-times ${ }^{12}$ forms the basic Lagrangian to which we add a Lagrangian for the EM field. We point out that earlier work by Amorim $^{7}$ and by de Ritis et al. ${ }^{6}$ using the self-consistent formalism assumes that the EM field is a two-form. Instead here, we assume that the EM field is minimally coupled to the geometry of RC space-time via the asymmetric connection of RC space-time. The EM Lagrangian contains the usual field Lagrangian plus an interaction term between the EM field and magnetic dipoles and a current term. ${ }^{13}$ It will be necessary to show how one is able to conserve gauge invariance, not only for the EM field tensor, but also for the Lagrangian as a whole.

Our treatment of a spinning fluid interacting with an EM field is closely related to that of the EinsteinMaxwell theory in Riemannian geometry in which the flat space derivatives of Minkowski space are generalized via the Riemannian connection to the covariant derivatives of Riemannian geometry. As we noted earlier, it is purely an accident, due to the symmetry of the Christoffel connection, that this approach in GR coincides with the two-form approach for the electromagnetic field. In our self-consistent approach, all fields, including the electromagnetic field, will be coupled to the geometry via the RC connection. The EM fields are thus considered part of the system and therefore mutually interact with all fields. By contrast, the two-form approach seems to treat the EM field as an external field.

In Sec. II, we briefly review the self-consistent Lagrangian for a perfect fluid with spin density, and in Sec. III, we introduce the EM Lagrangian. We discuss gauge invariance in Sec. IV. The complete Lagrangian and its variation is given in Sec. V, and we derive the various field equations in Sec. VI. In Sec. VII, we demonstrate several of the technical details involving the field equations such as charge conservation, spin transport, and the interaction between the EM field and the spin energymomentum density. We give our conclusions in Sec. VIII.

\section{LAGRANGIAN FOR PERFECT FLUID WITH SPIN DENSITY}

The self-consistent Lagrangian formulation for perfect fluids with spin density has been described for both general relativity and RC space-times. ${ }^{12,14}$ In these spacetimes, a physical property of matter-the spin density-is coupled to a natural geometric object-the proper torsion-of space-time ${ }^{15}$ via the torsion field equation. The Lagrangian density is given by

$$
L=L_{G}+L_{S}
$$

where the Lagrangian densities for the gravitational field, spinning fluid, and EM field are given, respectively, by

$$
L_{G}=e\{\Re / 2 \kappa\},
$$

and

$$
\begin{aligned}
L_{S}= & e\left\{-\rho\left[1+\epsilon\left(\rho, s, s_{i j}\right)\right]-\lambda_{2} \stackrel{*}{\nabla}_{i}\left(\rho u^{i}\right)+\lambda_{3} u^{k} \partial_{k} X\right. \\
& +\lambda_{4} u^{k} \partial_{k} s+\lambda_{1}\left(g_{i j} u^{i} u^{j}+1\right)-k \rho a^{1 i} \dot{a}_{i}^{2} \\
& +\lambda_{11}\left(g_{i} \rho^{1 i} a^{1 j}-1\right)+\lambda_{22}\left(g_{i j} a^{2 i} a^{2 j}-1\right) \\
& \left.+2 \lambda_{12} g_{i} a^{1 i} a^{2 j}+2 \lambda_{14} g_{i j} a^{1 i} u^{j}+2 \lambda_{24} g_{i} a^{2 i} u^{j}\right\},
\end{aligned}
$$

where $\Re$ is the scalar curvature, $\kappa=8 \pi G, G$ is the gravitational constant, $g_{i j}$ is the metric tensor with holonomic indices given by latin characters such that $i=0,1,2,3$, and $e=\sqrt{-g}$, where $g=\operatorname{det}\left(g_{i j}\right)$. The matter density is $\rho, s$ is the entropy density, $X$ is the fluid parameter associated with the Lin constraint, ${ }^{16}$ and $a^{\mu i}$ are tetrads with anholonomic indices $\mu=1,2,3,4$ such that $a^{4 i}=u^{i}$ is the fourvelocity of the fluid particles. The function $\epsilon$ then represents the internal energy of the fluid that is a function of the density, entropy, and the spin of the fluid in the selfconsistent formalism. The various $\lambda$ 's are the Lagrange multipliers that ensure particle number conservation, Lin constraint, conservation of entropy, and the orthonormality of the tetrads. An overdot represents the action of derivatives along fluid flow lines, $u^{k} \nabla_{k} a^{2 i} \equiv \dot{a}^{2 i}$, where $\nabla_{k}$ is the covariant derivative in RC space-time. The "star" derivative, $\stackrel{*}{\nabla}_{i}=\nabla_{i}+2 S_{i}$, where $S_{i}=S_{i x}{ }^{x}$ is the torsion vector. A discussion of the star derivative is given by Hehl. ${ }^{17}$

The first five terms in the spin Lagrangian are the generalization to RC space-time of the general relativistic perfect fluid Lagrangian density of Ray. ${ }^{18}$ The remaining terms give the generalization of Halbwachs' special relativistic treatment of spinning fluids ${ }^{19}$ to $\mathrm{RC}$ space-times. ${ }^{20}$ The spin tensor is

$$
s^{i j}=k\left(a^{1 i} a^{2 j}-a^{1 j} a^{2 i}\right) .
$$


In the self-consistent formulation, the thermodynamics of a spinning fluid is described by

$$
d \epsilon=T d s-p d(1 / \rho)+\frac{1}{2} w_{i j} d s^{i j},
$$

where $T$ is the temperature, $p$ is the pressure, and $w_{i j}=\dot{a}_{i}^{u} a_{\mu j}$ is the tetrad angular velocity. After introducing the EM Lagrangian in the next section, we will generalize the thermodynamics to include EM contributions to the internal energy.

\section{LAGRANGIAN FOR CHARGED, SPINNING FLUID WITH MAGNETIC DIPOLES}

We wish to extend the general form of the EM Lagrangian given earlier by Amorim, ${ }^{13}$

$$
L_{\mathrm{EM}}=e\left\{-\frac{1}{4} F^{i j} F_{i j}+\frac{1}{2} M^{i j} F_{i j}+J^{k} A_{k}\right\},
$$

where $F^{i j}$ is the EM field tensor, $M^{i j}=\rho \chi s^{i j}$ is the magnetization tensor, $\chi$ is the magnetic susceptibility, $J^{k}=q \rho u^{k}$ is the current four-vector, $q$ is the electronic charge, and $A_{k}$ is the four-vector potential of the EM field. However, we do not assume that the EM field tensor is a two-form. Instead we minimally couple the EM field to the geometry, in the sense that

$$
F_{i j}=2 \nabla_{[i} A_{j]},
$$

where $\nabla_{i}$ is the covariant derivative in RC space-time.

If the EM field is coupled to the geometry of spacetime, then it, as well, should be treated as a thermodynamic variable in the self-consistent formulation. The thermodynamics of magnetic systems has been discussed extensively by Guggenheim. ${ }^{21}$ The Hamiltonian dynamics of neutral EM fluids with induction is described by Holm. ${ }^{22}$ Accordingly, for charged perfect fluids with spin density, we extend the thermodynamics from Eq. (8) to

$d \epsilon=T d s-p d \frac{1}{\rho}+\frac{1}{2} w_{i} d s^{i j}+\frac{1}{2} m^{i j} d F_{i j}-\frac{1}{2 \rho} F^{i j} d F_{i j}$

where $m^{i j}=\chi s^{i j}$. The particular form of the magnetization term is similar to the form given by Sychev. ${ }^{23}$ The interaction term for a dipole, $\mathrm{m}^{i j}$, was earlier included in charged spin fluids by Amorim ${ }^{7}$ and de Ritis et al ${ }^{6}$ However, the field energy involving only $F^{i j}$ was not included. Without this latter term, the dipole energy arises only from interactions with external fields, neglecting the dipole-dipole interaction energy. By including the last term in Eq. (11), we include the energy density of all EM fields, including the dipole fields, as part of the total system.

In the next section, we show how we must augment the EM Lagrangian in order to maintain gauge invariance.

\section{GAUGE INVARIANCE}

The idea behind gauge invariance in any Lagrangian based theory follows the general approach for the Dirac Lagrangian. For Dirac ficlds, gauge invariance requires extending the usual gauge transformation of the vector potential to include a "gauge transformation of the second kind" 24 for the quantum Dirac field. The equivalent field in RC space-time is the torsion field. Thus we require that $F^{i j}$ (as well as the total Lagrangian, which we will discuss in Sec. V) be invariant under the extended gauge transformation ${ }^{25}$

$$
\begin{aligned}
& A_{j}^{\prime}=A_{j}-\partial_{j} \varphi, \\
& S_{i j}^{\prime k}=S_{i j}^{k}+\frac{2}{3} b \delta_{[i}^{k} \partial_{j]} \varphi,
\end{aligned}
$$

where $\varphi(x)$ is the gauge field and $b$ is a constant of proportionality. The equivalent effect on the connection is

$$
\Gamma_{i j}^{\prime k}=\Gamma_{i j}^{k}+\frac{2}{3} b \delta_{i}^{k} \partial_{j} \varphi-\frac{2}{3} b g_{i j} \partial^{k} \varphi .
$$

Previous attempts to generalize the Dirac field to RC space-time only seems to have been successful providing the torsion field is restricted to the torsion vector part ${ }^{9,11}$ without proper torsion. Such a restriction, in our case, would mean that our matter Lagrangian could only be a perfect fluid without spin density since the proper torsion is directly related to the spin density. ${ }^{15}$ However, we do note that the torsion vector seems to play an important role in these theories, and in some cases is proportional to the vector potential itself. ${ }^{10}$ In order to understand this aspect of our Lagrangian, we write the torsion tensor in terms of its proper (trace-free) and torsion vector (trace) parts

$$
S_{i j}^{k}=\widehat{S}_{i j}^{k}-\frac{2}{3} \delta_{[i}^{k} S_{j]}
$$

where the overcaret indicates the proper torsion part and $S_{j} \equiv S_{j k}{ }^{k}$ defines the torsion vector. Combining Eqs. (13) and (15), we see immediately that the extended gauge transformation of the torsion tensor is equivalent to the transformation

$$
\begin{aligned}
& S_{j}^{\prime}=S_{j}-b \partial_{\rho}, \\
& \hat{S}_{i j}^{\prime k}=\hat{S}_{i j}{ }^{k} .
\end{aligned}
$$

By comparison with Eq. (12), one may conclude that the torsion vector and the vector potential are proportional, i.e.,

$$
S_{j} \equiv b A_{j}
$$

We will therefore assume this restriction in the EM Lagrangian. 
The combined action of the extended gauge transformation on the EM field gives

$F_{i j}^{\prime}=F_{i j}-2\left\{\frac{2}{3} b A_{[i} \partial_{j]} \varphi+\frac{2}{3} S_{[j} \partial_{i]} \varphi-\hat{S}_{i j}{ }^{k} \partial_{k} \varphi\right\}$

If the torsion vector is identified with the vector potential [Eq. (18)] and using the proper torsion field equation ${ }^{15}$

$$
\hat{S}_{i j}^{k}=(\kappa / 2) \rho s_{i j} u^{k},
$$

one finds that the transformed EM field is now invariant providing we further require that the gauge in the tetrad (comoving) frame of the spinning fluid is not an explicit function of time, i.e.,

$$
u^{0} \partial_{0} \varphi=0 .
$$

This is equivalent to the covariant constancy of $\varphi$ along fluid flow lines; however, by choice of the tetrads associated with the spin density given in Eq. (7), i.e., the comoving frame of the fluid, this reduces to the above condition on the gauge. Equation (20) is not assumed but comes from the Lagrangian variation. We show this in Sec. VI. The condition on the gauge, Eq. (21), is very similar to the harmonic condition imposed in classical electromagnetism when, for example, one imposed a particular gauge such as the Lorentz gauge. The same effect occurs for general relativity. ${ }^{26}$ Thus we are led naturally to the addition of a gauge fixing term to our EM Lagrangian. $^{26-28}$

Because of the above considerations, we could add a term to the EM part of the Lagrangian that fixes the Lorentz gauge in RC space-times, but first we must determine whether we can also make the total Lagrangian invariant under gauge transformations as well. It is not difficult to determine that the perfect fluid with the spin part of the Lagrangian is invariant, but the gravitational field part is not. It is instructive to write the scalar curvature in terms of its Riemannian plus torsion parts,

$\Re=K+4 \nabla_{k}^{\{\}} S^{k}-\frac{8}{3} S_{k} S^{k}+\hat{S}_{j k} \widehat{S}^{j k l}+2 \widehat{S}_{j k} \hat{S}^{j l k}$,

where the scalar curvature $K$ and the covariant derivative $\nabla_{k}^{\{\}}$are those of Riemannian space-time. Considering only the torsion vector terms, we note that the variation of the divergence term with respect to torsion vanishes, but there is the contribution from the torsion vector squared term

$$
\delta\left\{-\frac{8}{3} S_{k} S^{k}\right\}=-\frac{16}{3} S_{k} \delta S^{k},
$$

in order to maintain gauge invariance of the total Lagrangian, the correct form of the gauge fixing term should then be

$$
L_{\mathrm{GF}}=-e\left\{(2 b / 3 \kappa) \nabla_{k} A^{k}\right\} .
$$

Our form of the EM Lagrangian is found by combining Eqs. (9) and (24) plus a (torsion vector)-(vector potential) constraint term following Eq. (18),

$$
\begin{aligned}
L_{E}= & e\left\{-\frac{1}{4} F^{i j} F_{i j}+\frac{1}{2} M^{i j} F_{i j}+J^{k} A_{k}+C^{k}\left(S_{k}-b A_{k}\right)\right. \\
& \left.-\frac{2 b}{3 k} \nabla_{k} A^{k}\right\} .
\end{aligned}
$$

In the next section we find the variation of the total Lagrangian.

\section{VARIATION OF LAGRANGIAN FOR CHARGED, SPINNING FLUID IN RC SPACE-TIME}

The total Lagrangian for a charged perfect fluid with spin density is the combination of Lagrangians given by Eq. (5), (6), and (25),

$$
\begin{aligned}
L=L_{G}+L_{F}+L_{E}= & e\left[\frac{\Re}{2 \kappa}-\rho\left[1+\epsilon\left(\rho, s, s_{i j} F_{i j}\right)\right]-\lambda_{2}{ }^{*} \nabla_{i}\left(\rho u^{i}\right)+\lambda_{3} u^{k} \partial_{k} X+\lambda_{4} u^{k} \partial_{k} s-k \rho a^{1 i} \dot{a}^{2}{ }_{i}+\lambda_{1}\left(g_{i j} u^{i} u^{i}+1\right)\right. \\
& +\lambda_{11}\left(g_{i j} a^{1 i} a^{1 j}-1\right)+\lambda_{22}\left(g_{i j} a^{2 i} a^{2 j}-1\right)+2 \lambda_{12} g_{i} a^{1 i} a^{2 j}+2 \lambda_{14} g_{i j} a^{1 i} u^{j}+2 \lambda_{24} g_{i j} a^{2 i} u^{j}-\frac{1}{4} F^{i j} F_{i j} \\
& \left.+\frac{1}{2} M^{i j} F_{i j}+J^{k} A_{k}-\frac{2 b}{3 \kappa} \nabla_{k} A^{k}+C^{k}\left(S_{k}-b A_{k}\right)\right\}
\end{aligned}
$$

where the variables of variation are $g_{i j}, \hat{S}_{i j}{ }^{k}, S_{k}, \rho, s, X, a^{1 i}, a^{2 i}, u^{i}, A_{k}$, the various Lagrange multipliers, $\lambda$, and $C^{k}$. In order to complete the variation of $L$, we must add to this system the thermodynamic relationship for a charged spinning fluid given by Eq. (11).

The total Lagrangian [Eq. (26)] yields the following variational field equations, $\delta L$ : 
metric equation $\delta g_{i j}$

$$
\begin{aligned}
\frac{1}{2 \kappa}[ & \left.-G^{(i j)}+\vec{\nabla}_{k}\left(T^{k i j}+T^{k i j}\right)\right]-\frac{1}{2} \rho(1+\epsilon) g^{i j}-\frac{1}{2} \rho k a^{1 l} \dot{a}_{l}^{2} g^{i j}+\stackrel{*}{\nabla}_{l}\left[\rho k u^{l} a^{(1 i}\right] a^{2 j)}-\frac{1}{2} \stackrel{\nabla}{*}_{k}\left[\rho s^{k(i} u^{j}\right)-\frac{1}{2} \nabla_{l}^{*}\left[\rho u^{l} k a^{(1 i} a^{2 j)}\right] \\
& +\frac{1}{2} \rho \dot{\lambda}_{2} g^{i j}+\lambda_{1} u^{i} u^{j}+\lambda_{12} a^{(1 i} a^{2 j)}+\lambda_{11} a^{1 i} a^{1 j}+\lambda_{22} a^{2 i} a^{2 j}+2 \lambda_{14} u^{(i} a^{1 j)}+2 \lambda_{24} u^{(i} a^{2 j)}-\frac{1}{2}\left[F^{l i} F_{l}^{j}+\frac{1}{4} g^{i j} F^{k l} F_{k l}\right] \\
& +\frac{1}{4} M^{k l} F_{k l} g^{i j}-\frac{4 b}{3 \kappa}\left[S^{(l} A^{j)}-\frac{1}{2} g^{i j} A S^{l}\right]=0,
\end{aligned}
$$

where $T_{i j}{ }^{k}=S_{i j}{ }^{k}+2 \delta_{[i}^{k} S_{j]}$ is the modified torsion tensor. ${ }^{29}$ Proper torsion $\delta \hat{S}_{i j}^{k}$,

$$
\left.-(1 / \kappa)\left[\hat{S}_{k}^{i j}-\widehat{S}_{k}^{i j}+\hat{S}_{k}^{i}\right]+\left[\rho u^{[i} s_{k}\right]_{k}-\frac{1}{2} \rho u_{k} s^{i j}\right]=0 ;
$$

torsion vector ${ }^{30} \delta S_{k}$,

$$
-(8 / 3 \kappa) S^{k}+C^{*}+(4 b / 3 \kappa) A^{k}=0
$$

vector potential $\delta A_{k}$,

$$
\begin{aligned}
& M^{i j} S_{i j}{ }^{k}-F^{i j} S_{i j}{ }^{k}+\stackrel{*}{\nabla}_{i} F^{i k}-\nabla_{i}^{*} M^{i k}-b C^{k}+(4 b / 3 \kappa) S^{k} \\
& \quad+q \rho u^{k}=0
\end{aligned}
$$

mass density $\delta \rho$,

$-\left(1+\epsilon+\frac{p}{\rho}\right)-\frac{1}{\rho} T_{S}+\dot{\lambda}_{2}+\frac{1}{2 \rho} M^{i j} F_{i j}+q u^{k} A_{k}=0$,

where $T_{S}$ is the spin kinetic energy.

Four-velocity $\delta u^{k}$,

$$
\begin{gathered}
-\rho k a^{1 i} \nabla_{k} a_{i}^{2}+\rho \nabla_{k} \lambda_{2}+\lambda_{3} \partial_{k} X+\lambda_{4} \partial_{k} s+2 \lambda_{1} u_{k} \\
+2 \lambda_{14} a_{k}^{1}+2 \lambda_{24} a_{k}^{2}+q \rho A_{k}=0
\end{gathered}
$$

tetrads,

$$
\begin{aligned}
& \delta a^{1 i}: \quad-\rho k w_{i j} a^{2 j}-\rho k \dot{a}^{2}{ }_{i}+2 \lambda_{11} a^{1}{ }_{i}+2 \lambda_{12} a^{2}{ }_{i}+2 \lambda_{14} u_{i} \\
& \quad+\rho \chi k F_{i j} a^{2 j}=0, \\
& \delta a^{2 i}: \quad-\rho k w_{j i} a^{1 j}+\rho k a^{1}{ }_{i}+\rho k \dot{a}_{i}{ }_{i}+2 \lambda_{22} a^{2}{ }_{i}+2 \lambda_{12} a^{1}{ }_{i} \\
& \quad+2 \lambda_{24} u^{i}+\rho \chi k F_{j} a^{1 j}=0
\end{aligned}
$$

Lin $\delta X$,

$$
\stackrel{*}{\nabla}_{k}\left(\lambda_{3} u^{k}\right)=0
$$

entropy $\delta s$,

$$
\stackrel{*}{\nabla}_{k}\left(\lambda_{4} u^{k}\right)+\rho T=0 .
$$

The Lagrange multipliers give the following constraint equations:

Conservation of particle number $\delta \lambda_{2}$,

$$
\stackrel{*}{\nabla}_{i}\left(\rho u^{i}\right)=0 ;
$$

Lin constraint $\delta \lambda_{3}$,

$$
\dot{X}=0
$$

conservation of entropy $\delta \lambda_{4}$,

$$
\dot{s}=0
$$

and

orthonormality of tetrads $\delta \lambda_{\mu v}$,

$$
g_{i} a^{\mu i} a^{v j}=\eta^{\mu \nu}
$$

where $\eta^{\mu \nu}$ is the Minkowski metric, with signature $(1,1,1,-1)$, and $\lambda_{44} \equiv \lambda_{1}$ so that the four-velocity constraint is contained in Eq. (40) as well:

torsion constraint $\delta C^{k}$,

$$
S_{k}=b A_{k} \text {. }
$$

Equations (27-41) represent the raw variational field equations obtained from $L$. In the next section, we arrive at the useful form of the metric, torsion, and EM field equations.

\section{FIELD EQUATIONS FOR A CHARGED SPIN FLUID}

\section{A. Field equations for spin}

The proper torsion field equation is obtained from Eq. (28) by taking the cyclic permutation of the indices $(k i j) \rightarrow(i j k)$ and then adding to the original. We find 


$$
\widehat{S}_{i k}^{k}=(\kappa / 2) \rho s_{i j} u^{k}
$$

which is identical with Eq. (20), which we had assumed earlier in our discussions in Sec. IV on gauge invariance. It is also in agreement with earlier work on the status of the proper torsion for spinning fluids in RC space-time, ${ }^{15}$ which establishes the relationship between the spin density of a fluid and torsion.

In order to reduce the metric equation (27) to a more recognizable form, we must first replace the tetrads by tensors and substitute for the Lagrange multipliers.

Multiplying the $\delta a^{1 i}$ equation (33) by $a^{2 i}$, we find that

$$
\lambda_{12}=0 \text {. }
$$

Then multiplying the $\delta a^{2 i}$ equation (34) by $a^{1 i}$ and using the above relationship, we obtain

$$
\dot{k}=0 \text {, }
$$

which says that the spin module function is covariantly constant along fluid flow lines. This just says that the spin in the tetrad (anholonomic) frame is a constant. ${ }^{20}$

\section{B. Stress energy content}

Multiplying the $\delta a^{1 i}$ equation (33) by $a^{1 i}$ and the $\delta a^{2 i}$ equation (34) by $a^{2 i}$, antisymmetrizing $i j$, using Eqs. (43) and (44), we find that

$$
\lambda_{11}=\lambda_{22}=T_{S}-\frac{1}{4} M^{i j} F_{i j}
$$

where $T_{S}=\frac{1}{2} \rho w_{i S^{j}}{ }^{i j}$ is the spin kinetic energy. Also, multiplying the $\delta \rho$ equation (31) by $\rho$, we find

$\rho \lambda_{2}=\rho(1+\epsilon+p / \rho)+T_{S}-\frac{1}{2} M^{i j} F_{i j}-q \rho u^{k} A_{k}$.

Then multiplying the $\delta u^{k}$ equation (32) by $u^{k}$ and substituting for $\lambda_{2}$, we get

$$
\lambda_{1}=\frac{1}{2} \rho(1+\epsilon+p / \rho)-\frac{1}{4} M^{i j} F_{i j}
$$

If we multiply the $\delta a^{1 i}$ equation (33) by $u^{i}$, we find

$$
\lambda_{14}=\rho k \dot{u} a^{2 j}+\frac{1}{2} \rho \chi k F_{i j} u^{i} a^{2 j},
$$

and similarly, $u^{i}$ times the $\delta a^{2 i}$ equation (34) gives

$$
\lambda_{24}=-\rho k \dot{u} a^{1 i}-\frac{1}{2} \rho \chi k F_{i j} u^{i} a^{1 j} .
$$

Multiplying the first of these two equations by $u^{(i} a^{1 j)}$, the second by $u^{(i} a^{2 j)}$, and then adding gives the identity

$\lambda_{14} u^{(i} a^{1 j)}+\lambda_{24} u^{(i} a^{2 j)}=\rho u^{\left(i s^{j}\right) k} \dot{u}_{k}+\frac{1}{2} \rho u^{l} F_{l k} u^{(i} m^{j) k}$.

A necessary relation is obtained from the $\delta a^{1 i}$ and $\delta a{ }^{2 i}$ variations by multiplying the first by $a^{1 j}$ and the second by $a^{2 j}$, symmetrizing on ( $\left.i j\right)$, adding the two re- sulting equations, noting that $\lambda_{12}$ and $\dot{k}=0$, and finally substituting for $\lambda_{14}$ and $\lambda_{24}$ using the combination given by Eq. (50). After identifying the spin tensor, Eq. (7), we obtain the intermediate step

$$
\begin{aligned}
& \rho w_{k}^{\left(i s^{j}\right) k}+\rho k\left[\dot{a}^{(1 i} a^{2 j)}-a^{(1 j} a^{2 i)}\right]+2 \lambda_{11}\left[a^{(1 i} a^{1 j)}\right. \\
& \left.+a^{(2 i} a^{2 j)}\right]+2 \lambda_{14} u^{(i} a^{1 j)}+2 \lambda_{24} u^{(i} a^{2 j)} \\
& +\rho m^{k\left(j_{F_{k}}{ }^{i)}=0 .\right.}
\end{aligned}
$$

For convenience, we will introduce the Amorim tensor $^{7}$

$$
B_{k}^{j}=\left(\delta_{l}^{j}+u^{j} u_{l}\right) F_{k}^{l}
$$

We then divide Eq. (51) by two, add back Eq. (50), and substitute for the Amorim tensor to obtain the useful relationship

$$
\begin{aligned}
\lambda_{11}[ & \left.a^{(1 i} a^{1 j)}+a^{(2 i} a^{2 j)}\right]+2\left[\lambda_{14} u^{(i} a^{1 j)}+\lambda_{24} u^{(i} a^{2 j)}\right] \\
= & \rho u^{\left(i^{j}\right) k} \dot{u}_{k}-\frac{1}{2} \rho w_{k}{ }^{(i} s^{j) k}-\frac{1}{2} \rho m^{k(i} B^{j)} k \\
& \\
& -\frac{1}{2} \rho k\left[\dot{a}^{(1 i} a^{2 j)}-a^{(1 i} \dot{a}^{2 j}\right) .
\end{aligned}
$$

Finally, upon substituting Eqs. (37), (43), (46), (47), and (53) into Eq. (27), we finally obtain our form of the metric field equation

$$
G^{(i j)}-\vec{\nabla}_{k}\left(T^{k i j}+T^{k j i}\right)=\kappa T^{i j},
$$

where the symmetric energy-momentum tensor has the components

$$
T^{i j}=T_{M}^{i j}+T_{S}^{i j}+T_{\mathrm{EM}}^{i j}+T_{\mathrm{GF}}^{i j},
$$

with perfect fluid energy-momentum tensor

$$
T_{M}^{i j}=\rho\left[(1+\epsilon+p / \rho)-\frac{1}{2} m^{k l} F_{k l}\right] u^{i} u^{j}+p g^{i j},
$$

where we have included a magnetic energy term to the internal energy, with spin energy-momentum tensor

$$
T_{S}^{i j}=2 \rho u^{(i} s^{j) k} \dot{u}_{k}+\dot{\nabla}_{k}^{*}\left[\rho u^{\left(i s^{j) k}\right.}\right]-\rho w_{k}^{\left(i s^{j) k}\right.}
$$

an electromagnetic energy-momentum tensor

$$
T_{\mathrm{EM}}^{i j}=-\left[F^{k i} F_{k}^{j}+\frac{1}{4} g^{i j} F^{k l} F_{k l}+M^{k(i} B^{j)}{ }_{k}\right],
$$

plus a gauge fixing, energy-momentum correction tensor

$$
T_{\mathrm{GF}}^{i j}=-(8 / 3 \kappa)\left(S^{i} S^{j}-\frac{1}{2} g^{i j} S^{k} S_{k}\right) .
$$

Although this gauge fixing term seems surprising at first, its origin derives from the requirement of overall gauge invariance of the total Lagrangian. Writing out the left- 
hand side of Eq. (54) in terms of its Riemannian plus proper torsion and torsion vector parts,

$$
\begin{aligned}
& G^{(i j)}-2 \stackrel{*}{\nabla}_{k} T^{k(i j)}=G^{i j}(\{\})+2 \hat{S}^{(i}{ }_{l x} \hat{S}^{j) l x}+\hat{S}^{(i}{ }_{l x} \widehat{S}^{j) x l} \\
& -\hat{S}^{x l\left(i \hat{S}_{x} l\right)}-\frac{1}{2} g^{i j}\left(\hat{S}^{l m x} \hat{S}_{l m x}\right. \\
& \left.+2 \hat{S}^{l m x} \widehat{S}_{l x m}\right)-\frac{8}{3}\left(S^{i} S^{j}-\frac{1}{2} g^{i j} S_{k} S^{k}\right),
\end{aligned}
$$

we see that the gauge fixing terms exactly cancel the torsion vector part, leaving only the Einstein tensor plus spin-squared terms after using the proper torsion field $\mathrm{Eq}$. (42).

\section{Electromagnetic field equations}

Upon multiplying the $\delta S_{k}$ Eq. (29) by $b$, adding to the $\delta A_{k}$ Eq. (30), and simplifying the covariant derivatives, we find the EM field equation

$$
\nabla_{i}^{\{\}} F^{k i}=\nabla_{i}^{\{\}} M^{k i}+J^{k},
$$

where $\nabla_{i}^{[\}}$is the Riemannian covariant derivative.

The remarkable simplicity of the EM field Eq. (61) will be exploited in the next section in the discussion of charge conservation. We will also consider the effect of the EM field on spin transport and its interaction with spin energy momentum.

\section{PROPERTIES OF EM FIELD IN RC SPACE-TIME}

\section{A. Charge conservation}

From the form of $J^{k}=q \rho u^{k}$, we know a priori that $\stackrel{*}{\nabla}_{k} J^{k}=0$ because of the particle number conservation constraint Eq. (37). However, for any vector $V^{k}$ in RC space-time, it may be shown that

$$
\stackrel{*}{\nabla}_{k} V^{k}=\nabla_{k}^{\{\}} V^{k} .
$$

Then the divergence terms in Eq. (61) satisfy the condition (for example)

$\stackrel{*}{\nabla}_{k} \nabla_{i}^{\{\}} F^{k i}=\nabla_{\mid k}^{\{\}} \nabla_{l]}^{\{\}} F^{k i}=\frac{1}{2} K_{k i r}{ }^{k} F^{r i}+\frac{1}{2} K_{k i r}{ }^{i} F^{k r}=0$,

where $K_{i j k}^{l}$ is the Riemann tensor in Riemannian spacetime. Thus Eq. (61) under the action of the "star" derivative $\vec{\nabla}_{k}$ in $\mathrm{RC}$ space-time, satisfies the condition for charge conservative, even though the EM field, $F_{i j}$, contains explicit torsion terms. These same torsion terms in the EM field, however, lead to direct interaction of the EM field with spin transport and energy momentum.

\section{B. Spin transport}

In order to derive the spin transport equation, we can derive (in a similar manner) the antisymmetric equivalent to Eq. (51),

$$
\begin{aligned}
& \rho w_{k}{ }^{\left[i s^{j}\right] k}+\rho k\left\{\dot{a}^{[1 i} a^{2 j]}+a^{[1 i} \dot{a}^{2 j]}\right\} \\
& \quad+\left\{2 \lambda_{14} u^{[i} a^{1 j]}+2 \lambda_{24} u^{[i} a^{2 j]}\right\}-\rho F_{k}{ }^{[i} m^{j] k}=0,
\end{aligned}
$$

and the antisymmetric equivalent to Eq. (50),

$\lambda_{14} u^{[i} a^{1 j]}+\lambda_{24} u^{[i} a^{2 j]}=\rho u^{\left[i s^{j]}\right.} \dot{u}_{k}+\frac{1}{2} u^{l} F_{l k} u^{[i} m^{j] k}$.

From the definition of the parallel covariant derivative (dot derivative) on the spin tensor, we get

$$
\dot{s}^{i j} \equiv u^{k} \nabla_{k} s^{i j}=2 k\left\{\dot{a}^{[1 i} a^{2 j]}+a^{[1 i} \dot{a}^{2 j]}\right\},
$$

because $\dot{k}=0$. Combining Eqs. (64)-(66) and using the Amorim tensor, Eq. (52), we find the spin transport equation

$$
\dot{s}^{i j}+2 \dot{u}_{k} u^{[i} s^{j]}+m^{k[i} B^{j]}{ }_{k}=0 .
$$

The last term gives the torque term expected on a magnetic dipole in the presence of an EM field. If $B_{j k}$ vanishes, Eq. (67) reduces to the usual Fermi-Walker transport of the spin tensor. ${ }^{12}$

\section{Spin energy momentum}

We rewrite the spin energy-momentum tensor in terms of RC covariant derivatives that only contain proper torsion $\hat{\nabla}_{k}$ plus a remainder term. This gives

$$
T_{S}^{i j}=\widehat{T}_{S}^{i j}-\frac{4}{3} \kappa u^{\left(i_{S}^{j}\right) k} S_{k}=\widehat{T}_{S}^{i j}-\frac{4}{3} b \kappa u^{\left(i S^{j}\right) k} A_{k},
$$

where we have used the torsion constraint in the last step, and the overcaret represents terms independent of the torsion vector. In the absence of the EM field, i.e., $A_{k}=0$, then $S_{k}=0$ because of the torsion vector constraint Eq. (41). Thus $\widehat{T}_{\xi}^{i j}$ is the usual spin energy momentum of a perfect fluid with spin density when there is no EM field present. ${ }^{12}$ Thus the term in Eq. (68) containing the vector potential represents a direct interaction of the EM field with the spin. This alteration of the spin energy momentum is in addition to the usual energy shift due to a dipole term [the term containing $B^{j k}$ in Eq. (58) for $\left.T_{\mathrm{EM}}^{i j}\right]$. The extra interaction term in Eq. (68) does not occur if one assumes the two-form definition of the EM field tensor such as in the work of Amorim ${ }^{7}$ or de Ritis $e t$ $a l .{ }^{6}$ since the torsion vector vanishes. The direct source of this term is the thermodynamics for a spinning fluid in the presence of a torsion field. When there is no EM field, then particle number conservation is sufficient to remove the torsion vector. ${ }^{12}$ 


\section{Free field equations}

In part $\mathrm{A}$ of this section, we showed that the EM field satisfies the usual condition for charge conservation. To complete the discussion of the EM field, we need to consider the constraints on the potentials obtained from the dual field equations for Div B and Curl E, i.e., the free field equations. In an inertial frame these equations take the usual simple form, Div $\mathrm{B}=0$ and Curl $\mathrm{E}=-\partial B / \partial t$. Ellis $^{31}$ has shown that in systems with vorticity, the "...motion of a family of observers affects the form of Maxwell's equations [that] they would observe;...," including the free field equations. In a spin fluid, the torsion of the spin fluid contributes directly to the vorticity. ${ }^{32}$ Thus there will be contributions to the free field equations due to the intrinsic spin density of the fluid. In order to see this, we write the dual field

$$
{ }^{*} F^{i j}=\frac{1}{2} \epsilon^{i j k l} F_{k l}
$$

where $e^{i j k l}$ is the totally antisymmetric Levi-Civita tensor. ${ }^{4}$ For consistency with Eq. (63), we take the "star" divergence of Eq. (69),

$$
\stackrel{*}{\nabla}_{j}^{*} F^{i j}=\nabla_{j}^{\{\}^{*}} F^{i j}+{ }^{*} F^{k j} \hat{S}_{j k}{ }^{x}=\hat{\nabla}_{j}^{*} F^{i j}
$$

where the overcaret on $\hat{\nabla}_{j}$ refers to the covariant derivative constructed from the RC connection, $\hat{\Gamma}_{i j}^{k}$, which contains only proper torsion (i.e., no torsion vector). Also, because of the torsion vector-vector potential constraint term in the EM Lagrangian Eq. (25), then

$$
F_{i j}=2 \nabla_{[i} A_{j]}=2 \widehat{\nabla}_{[i} A_{j]}=\widehat{F}_{i j},
$$

so that

$$
{ }^{*} F^{i j}={ }^{*} \widehat{F}^{i j}
$$

as well. Substituting Eq. (69) into Eq. (70) and using Eqs. (71) and (72), we obtain

$$
\begin{aligned}
& \vec{\nabla}_{j}^{*} F^{i j}=\hat{\nabla}_{j}^{*} \widehat{F}^{i j}=e^{i j k l} \widehat{\nabla}_{[j} \hat{\nabla}_{k} A_{l]} \\
& =e^{i j k l}\left[-\frac{1}{2} \hat{R}_{[j k l]}^{x} A_{x}-\hat{\nabla}_{x} A_{[l} \hat{S}_{j k]}^{x}\right] \\
& =e^{i j k l}\left[-\hat{\nabla}_{[j} \hat{s}_{k l]}^{x} A_{x}-\hat{\nabla}_{x} A_{[l} \hat{S}_{j k]}^{x}\right],
\end{aligned}
$$

where, in the last step, we have used the circulating identity for the curvature tensor. ${ }^{33}$ The form of Eq. (73) explicitly shows how the torsion mimics the effect of frame dependence described by Ellis. Both the vorticity effect described by Ellis and the spin density effect described here are due to matter distributions. Note that if the spin density vanishes, one obtains the usual free field equations.

\section{CONCLUSIONS}

We have shown that it is possible to find a variational theory in RC space-times for a magnetized charged perfect fluid with spin density in which the EM field is minimally coupled to the geometry through the RC connection [see Eq. (10)]. We require that the total Lagrangian for the theory be gauge invariant under the extended gauge transformation given by Eqs. (12) and (13). This necessarily implies that the torsion vector be proportional to the EM vector potential plus the addition of a gauge fixing term to the total Lagrangian. The gauge must be time independent in the comoving frame of the spin fluid.

With the above restrictions, we find that the field equation for the Maxwell field takes the same form as in the Einstein-Maxwell theory in Riemannian space-time in theories that do not assume a minimal coupling approach. In our development, however, the field tensor $F^{i j}$ in Eq. (61) contains specific torsion terms, while both charge and particle number are conserved. Because the EM field specifically couples to the RC geometry, we find that the EM field directly influences the spin energy momentum in addition to the usual dipole-EM field energy contribution. The theory does not neglect the interaction of the EM field with the spin density distribution and the consequent change in spin energy momentum. The origin of this is in the extended thermodynamics given by Eq. (11). We have also shown that the spin density affects the free field equations due to the torsion contributions to the vorticity in RC space-time. The same effect has been described by Ellis ${ }^{31}$ for systems with vorticity in GR.

The spin tensor obeys a spin transport equation that includes a term describing the torque of a magnetic dipole in the presence of an EM field. When the EM field vanishes, the spin transport equation becomes the usual Fermi-Walker transport.

The metric field equation gives a symmetric energy momentum tensor that now includes an internal energy term due to the energy of a magnetic dipole in the presence of an EM field; a symmetric spin energy-momentum tensor (discussed above); a symmetric EM energy-momentum term that includes a dipole term involving the Amorim tensor ${ }^{7}$ given in Eq. (52); and a (symmetric) gauge fixing term.

We have demonstrated a new way in which one can couple the EM field to RC space-times while retaining many of the expected properties of the EM field in the presence of a gravitational field. Our particular method treats the EM field as part of the overall system instead of as an external field, as in developments where the EM field is a two-form. We have a well defined theory of the interaction of the EM field with a perfect fluid with spin density in RC space-time that has as its limit the Lagrangian based theory of a spinning fluid in RC spacetime when the EM field vanishes.

There are many problems that can be studied with 
the extended stress energy tensor. The most natural area of application is to systems in which the EM field is embedded in the matter distribution itself. Examples of such systems are galactic and star forming matter clouds with differential rotation or the gravitational-EM description of condensed objects with strong gravitational and EM fields. Another interesting possibility is an application to ferromagnetofluids or low temperature superfluids with spin density. ${ }^{34}$

\section{ACKNOWLEDGMENTS}

The authors gratefully recognize the essential contributions of A. J. Fennelly and J. R. Ray to the importance and our understanding of the free field equations for the electromagnetic field.

${ }^{1} \mathrm{M}$. Novello, Phys. Lett. A 59, 105 (1976).

${ }^{2}$ R. Spinosa, Phys. Lett. A 125, 228 (1987).

${ }^{3}$ D. W. Sciama, in Recent Developments in General Relativity (Pergamon, Oxford, 1962), p. 415; Rev. Mod. Phys. 36, 463, 1103 (1964); T. W. B. Kibble, J. Math. Phys. 2, 212 (1961).

${ }^{4}$ C. W. Misner, K. S. Thorne, and J. A. Wheeler, Gravitation (Freeman, San Francisco, CA, 1973), Chap. 4.

${ }^{5}$ F. W. Hehl, P. von der Heyde, and G. D. Kerlick, Rev. Mod. Phys. 48, 393 (1976).

${ }^{6}$ R. de Ritis, M. Lavorgna, G. Platania, and C. Stornaiola, Phys. Rev. D 31, 1854 (1985).

${ }^{7}$ R. Amorim, Phys. Rev. D 31, 3099 (1985); Phys. Lett. A 104, 259 (1984).

${ }^{8}$ V. De Sabbata and M. Gasperini, Phys. Rev. D 23, 2116 (1981).

'S. Hojman, M. Rosenbaum, M. P. Ryan, and L. C. Shepley, Phys. Rev. D 17, 3141 (1978).

${ }^{10}$ R. T. Hammond, Gen. Relat. Grav. 20, 813 (1988); Class. Quantum. Grav. 6, L195 (1989).

"C. Mukku and W. A. Sayed, Phys. Lett. B 82, 382 (1979).

${ }^{12}$ J. R. Ray and L. L. Smalley, Phys. Rev. D 27, 1381 (1983); Phys.
Rev. Lett. 49, 1059 (1982); 50, 626E (1983).

${ }^{13}$ See, for example, R. Amorim, Phys. Lett. A 104, 259 (1984).

14 J. R. Ray, L. L. Smalley, and J. P. Krisch, Phys. Rev. D 35, 3261 (1987).

${ }^{15}$ L. L. Smalley and J. R. Ray, Gen. Relat. Grav. 18, 549 (1986).

${ }^{16}$ See, for example, J. R. Ray, J. Math. Phys. 13, 1451 (1972).

${ }^{17}$ F. W. Hehl, Gen. Relat. Grav. 4, 333 (1973); 5, 491 (1974)

${ }^{18}$ J. R. Ray, J. Math. Phys. 13, 1451 (1972).

${ }^{19}$ F. Halbwachs, Theorie Relativiste Des Fluides A Spin (Gauthier-Villar, Paris, 1960).

${ }^{20}$ For a discussion of the relative signs of the terms in the Lagrangian density, see L. L. Smalley and J. R. Ray, Phys. Lett. A 134, 87 (1988); Class. Quantum Grav. 7, 1445 (1990).

${ }^{21}$ E. A. Guggenheim, Proc. R. Soc. London Ser. A 20, 49 (1936); 20, 70 (1936).

${ }^{22}$ D. D. Holm, Physica D 25, 261 (1987).

${ }^{23}$ V. V. Sychev, Complex Thermodynamic Systems (Consultants Bureau, New York, 1973); see Eq. (3.17a), p. 56.

${ }^{24}$ A. Messiah, Quantum Mechanics (North-Holland, Amsterdam, 1962), Vol. II, p. 918.

${ }^{25}$ L. L. Smalley, Phys. Rev. D 33, 3590 (1986).

${ }^{26}$ D. Grensing and G. Grensing, Phys. Rev. D 28, 286 (1983); Gen. Relat. Grav. 15, 985 (1983).

${ }^{27}$ V. Fock, The Theory of Space Time and Gravitation (Pergamon, New York, 1959).

${ }^{28}$ P. A. M. Dirac, Phys. Rev. D 114, 929 (1959).

${ }^{29}$ I. M. Benn, Ann. Inst. Henri Poincaré 37, 67 (1982).

${ }^{30} \mathrm{As}$ an aid to those rederiving these equations, we write the equations including terms that cancel

$$
-\frac{8}{3 \kappa} S^{k}+C^{k}+\frac{4 b}{3 \kappa} A^{k}+\frac{2}{3}\left(M^{i k}-F^{i k}\right) A_{i}-\frac{2}{3}\left(M^{i k}-F^{i k}\right) A_{i}=0 .
$$

${ }^{31}$ G. R. F. Ellis, in Cargese Lectures in Physics, edited by E. Schatzman (Gordon and Breach, New York, 1973), Vol. 6, p. 13.

${ }^{32}$ A. J. Fennelly, J. P. Krisch, J. R. Ray, and L. L. Smalley, J. Math. Phys. 32, 485 (1991).

${ }^{33}$ J. A. Schouten, Ricci Calculus (Springer-Verlag, Berlin, 1954), 2nd ed.

${ }^{34}$ G. G. Asgekar and C. G. Patwardhan, Gen. Relat. Grav. 20, 289 (1988). 